\title{
Synthesis and characterization of Hydrogels based on Potato Starch/Poly(vinyl Alcohol)/N, N'- Methylenebisacrylamide
}

DIEGO DAVID PINZON-MORENO ( $\sim$ diegodpinzon@gmail.com )

Universidad Nacional Mayor de San Marcos https://orcid.org/0000-0003-1377-3101

Eder Clidio Vicuña-Galindo

Universidad Nacional Mayor de San Marcos

José Vulfrano Gonzáles-Fernández

Universidad Nacional Mayor de San Marcos

José Luis Soto-Gonzales

Universidad Nacional Mayor de San Marcos

María Verónica Carranza-Oropeza

Universidad Nacional Mayor de San Marcos

\section{Research Article}

Keywords: Hydrogel, potato starch, polyvinyl alcohol, N,N'-Methylenebisacrylamide, kinetic swelling and deswelling, thermal characterization, morphology characterization

Posted Date: July 19th, 2021

DOl: https://doi.org/10.21203/rs.3.rs-702429/v1

License: (9) (1) This work is licensed under a Creative Commons Attribution 4.0 International License.

Read Full License 


\title{
Synthesis and characterization of Hydrogels based on Potato Starch/Poly(vinyl Alcohol)/N, N'-Methylenebisacrylamide
}

Diego David Pinzón-Moreno ${ }^{1}$, Eder Clidio Vicuña-Galindo ${ }^{1}$, José Vulfrano GonzálesFernández ${ }^{1}$, José Luis Soto-Gonzales ${ }^{1}$, María Verónica Carranza-Oropeza ${ }^{1}$

\author{
${ }^{1}$ National University of San Marcos, Faculty of Chemistry and Chemical Engineering, Calle \\ Germán Amézaga No. 375, 15081, Lima, Peru \\ Idiegodpinzon@gmail.com \\ ORCID iD: 0000-0003-1377-3101 \\ Celular - WhatsApp: +51902461 751
}

\begin{abstract}
Several hydrogels were synthesized by free-radical polymerization in an aqueous medium based on potato starch (PS), poly(vinyl alcohol) (PVA), and N,N'Methylenebisacrylamide (MBAm), being possible to study these hydrogels as a function of the proportion of components incorporated. In this way, the products generated from the synthesis were characterized by swelling and deswelling kinetics, the first swelling being verified with Schott and statistical models, allowing to contrast the proximity between the experimental and theoretical behavior. Additionally, spectroscopy (FTIR), morphological (SEM), and thermal (TGA and DSC) analysis allowing to know the intrinsic characteristics of the material, increasing in general terms the knowledge of this type of material. In this context, it was possible to verify the characteristics and effectiveness of the synthesis and crosslinking of the main components. The experimental results obtained show that the synthesized hydrogels present representative first swellings consistent with kinetic and statistical models; however, there are significant changes in the second swelling derived from polymer degradation that occurs during the same swelling/deswelling cycles processes.
\end{abstract}

Keywords: Hydrogel, potato starch, polyvinyl alcohol, N,N'Methylenebisacrylamide, kinetic swelling and deswelling, thermal characterization, morphology characterization. 


\section{Funding Statement}

Authors are grateful to Concytec-Peru and The World Bank for the financial support of this project under the call "Mejoramiento y Ampliación de los Servicios del Sistema Nacional de Ciencia Tecnología e Innovación Tecnológica” 8682-PE, through Fondecyt grant 017-2019 FONDECYT BM INC.INV.

\section{Acknowledgment}

Special thanks and gratitude dedicated to Dr. J. Quispe and Dr. H. Loro for their valuable support, and to the Centro de Investigaciones Tecnológicas, Biomédicas y Medioambientales (CITBM), and the Laboratorio de Investigación en Control y Análisis de aguas, and the Laboratorio de Investigación en Nanotecnología de Alimentos of Jose Maria Arguedas National University.

\section{Conflict of interest}

All the authors declared no conflict of interest. 


\section{Introduction}

In recent years, hydrogels have been presented as a highly innovative material used for many practical purposes due to their well-known hydrophilic qualities. This hydrophilic capacity allows the retention and storage of liquids of different kinds, providing options that no other previously studied material had been able to offer. Currently, different types of hydrogels that have allowed their use in areas such as medicine, pharmacy, electronics, agriculture, purification, separation , and segregation of substances, applied biotechnology, among other areas of innovation and development, reasons for which their research related to the properties and applications still on a rise [1-5].

In this sense, PVA arouses interest in biotechnological areas due to its properties and characteristics such as its low degree of toxicity, biocompatibility, stability, economy, and can also be produced via non-petroleum routes; reasons for which the development of hydrogels based on PVA is attractive for noble purposes. In addition, another essential characteristic of this material is in the development of new materials such as composites, since PVA as a matrix favors the dispersity of colloids [6], allowing cost/benefit balances more in line with specific needs and friendly to the environment. The literature records various works on PVA-based hydrogels in conjunction with several types of natural or synthetic aggregates that allow obtaining materials with high development and application potential [1-2,7-10].

The use of aggregates of the natural origin (cellulose, saccharide, starch, etc.) have broad advantages because they are easy to acquire in multiple agro-industries, are environmentally friendly, are cheap, and allow optimization of some properties of polymer blends and hydrogels [11]. Among these renewable sources, starches are presented as a source of food and biomass present throughout the world and constant production during practically the entire period of the year [12]. These types of carbohydrates are found in significant amounts in many cereals, tubers, and roots kinds and are relatively easy to obtain [12-14].

Starch is constituted of two defined fractions, amylose and amylopectin, the latter being insoluble in water and with higher proportions in starch. This proportion varies according to the botanical source and its conditions [12,15], however, starch is mainly made up of amylose (20-30\%) and amylopectin (70-80\%) [16]. Among the main properties of interest of starch in the field of absorbent materials is the capacity for 
gelling/gelatinization, porosity, reversible absorption, swelling, and non-toxicity, attractive characteristics when formulating hydrogels of different types [13,16-20].

In this context, this work presents a complete study related to the synthesis and characterization of hydrogels based on PS, PVA, and MBAm as a crosslinking agent allowing the obtaining of absorbent materials. The characterization of the hydrogels was carried out employing experimental and theoretical analysis of the swelling kinetics, statistical study of the swelling performance, spectroscopic analysis of FTIR, morphological characterization utilizing SEM, and thermal analysis of the polymer through DSC and TGA, these characterizations allowed to determine fundamental properties for the best applicaction of this kind of polymer.

\section{Materials and methods}

\section{Materials}

Potato starch (PS) was obtained from HiMedia Laboratories, LLC, poly(vinyl alcohol) (PVA) from KHBoddin $\mathrm{GmbH}$, the N,N'-Methylenebisacrylamide (MBAm), and Potassium persulfate (KPS) was purchase from Central Drug House (P) Ltd - CDH. All materials were of analytical grade and used without further purification. On the other hand, distilled water was used for the hydrogel preparation and swelling measurements.

\section{Synthesis}

The amounts of reagents mentioned in Table 1 were pre-diluted in $40 \mathrm{ml}$ of distilled water; likewise, $1.5 \mathrm{~g}$ of KPS was diluted in the same amount of water for all the syntheses. The solutions were incorporated into a continuous stirred-tank reactor (CSTR) operated in mode batch with a capacity of $500 \mathrm{ml}$, with temperature, stirring, and atmosphere controlled, keeping the conditions of $70^{\circ} \mathrm{C}, 700 \mathrm{rpm}$, and nitrogen gas (N2) atmosphere constant throughout the reaction. The order of adding the solutions to the reactor started with the PS solution followed by the PVA, KPS and ending with the MBAm. Furthermore, the reagent addition was performed with equal time spans of 15 minutes and the synthesis time lasted 3 hours from the last addition of the MBAm solution. After this period the product was removed from the reactor, washed in a 50/50 solution of ethanol/distilled water, dried, ground, and sieved. 
Table 1 Matrix of components and its proportions

\begin{tabular}{ccrr}
\hline & \multicolumn{3}{c}{ Variables } \\
\cline { 2 - 4 } Sample & $\begin{array}{c}\text { PS } \\
(\boldsymbol{\%} \text { mass }) \\
\left(\boldsymbol{x}_{\mathbf{1}}\right)\end{array}$ & $\begin{array}{c}\text { PVA } \\
(\boldsymbol{\%} \text { mass }) \\
\left(\boldsymbol{x}_{\mathbf{2}}\right)\end{array}$ & $\begin{array}{c}\text { MBAm } \\
(\boldsymbol{\%} \text { mass }) \\
\left(\boldsymbol{x}_{\mathbf{3}}\right)\end{array}$ \\
\hline H1 & 35,00 & 50,00 & 15,00 \\
H2 & 92,00 & 5,00 & 3,00 \\
H3 & 47,00 & 50,00 & 3,00 \\
H4 & 77,75 & 16,25 & 6,00 \\
H5 & 49,25 & 38,75 & 12,00 \\
H6 & 71,75 & 16,25 & 12,00 \\
H7 & 80,00 & 5,00 & 15,00 \\
H8 & 55,25 & 38,75 & 6,00 \\
H9 & 63,50 & 27,50 & 9,00 \\
\hline
\end{tabular}

\section{Characterization}

Cyclic swelling and deswelling kinetic curves were obtained by periodically measuring the masses of the hydrogel immersed in water using the tea bag methodology at room temperature and subsequently calculated employing Equation 1. Once the different kinetic curves were obtained, such data was verified and compared utilizing pseudo-second-order swelling kinetics mathematical model, obtaining theoretical swelling parameters.

$$
\text { Swelling }(\%)=\frac{m_{s}-m_{d}}{m_{d}} * 100 \quad \text { (Equation 1) }
$$

where $m_{d}$ and $m_{s}$ represent the weights of the dried hydrogel and the hydrogel swelling to a defined time, respectively.

Based on the swelling results, the influence of the components in the synthesized hydrogel was analyzed. To this goal the study of the statistical relevance of components was continued, where ternary diagrams were constructed as a function of the components of PS, PVA, and MBAm as a function of equilibrium swelling in response, determining interesting regions for the production of this kind of hydrogel.

On the other hand, the hydrogels were characterized employing Fourier transform infrared spectroscopy - attenuated total reflectance (FTIR-ATR) in a Shimadzu IRTracer-100 spectrometer with the Pike MIRacle single reflection horizontal ATR accessory equipped with a ZnSe ATR crystal in transmittance mode. The wavenumber range from 300 to $4500 \mathrm{~cm}^{-1}$ at 64 scans and resolution at $4 \mathrm{~cm}^{-1}$ was used for the analysis.

The hydrogels were thermally characterized by Thermogravimetric Analysis (TGA) and Differential Scanning Calorimetry (DSC), these analyses were carried out in a TA Instruments TGA 550 and DSC 2500, respectively. TGA was carried out from 
room temperature to $600{ }^{\circ} \mathrm{C}$ under a heating rate at $10{ }^{\circ} \mathrm{C} / \mathrm{min}$ using nitrogen flow at $100 \mathrm{~mL} / \mathrm{min}$ as protective gas. For DSC was used nitrogen flow at $100 \mathrm{~mL} / \mathrm{min}$ as purge and protective, and the analysis temperatures setup were defined between -50 to $250{ }^{\circ} \mathrm{C}$ at a heating and cooling rate of $20{ }^{\circ} \mathrm{C} / \mathrm{min}$, the cooling stage was reached using liquid nitrogen.

Finally, Scanning Electron Microscopy (SEM) analyses were also carried out in FESEM, Hitachi Regulus 8230, Hitachi High-Tech Co. equipment, employing a secondary electron signal and voltage at $3,0 \mathrm{kV}$.

\section{Results and discussion}

The synthesis and subsequent processes allowed to obtain a series of hydrogels based on PS, PVA, and MBAm that have a gelatinous, yellowish, and soft texture in their wet state; already in their dry state the hydrogels turn pale and rigid. In Fig. 1 it is possible to observe some hydrogels obtained.
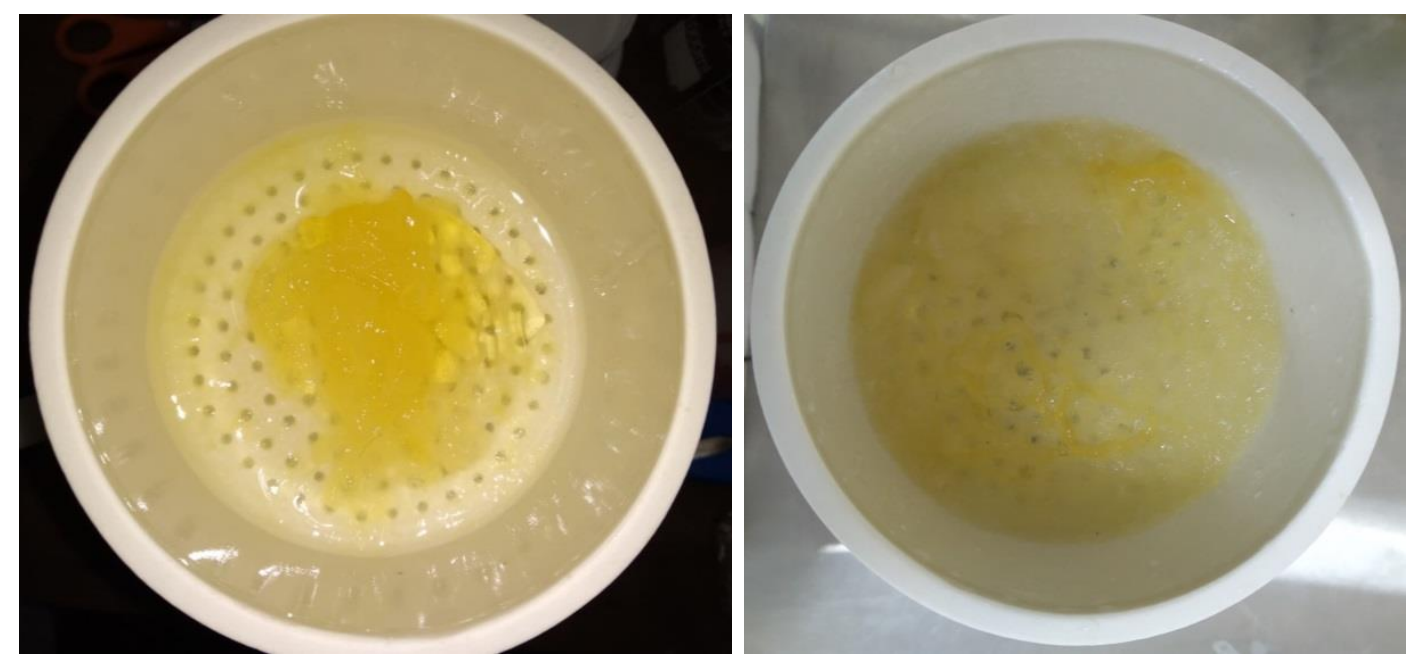

Fig. 1 Some hydrogels in the wash process

\section{Swelling and deswelling test}

Fig. 2 shows the results obtained regarding the kinetic curves of two consecutive swellings process of the hydrogels. As expected in a first swelling (Fig. 2A) the behavior describes a logarithmic function adjusting asymptotically to a value that represents an experimental equilibrium swelling $\left(W t_{1}\right)$ that depends on the composition of each hydrogel. Already in the second swelling curves (Fig. 2B), some transformations in the ideal swelling behavior are observed, partially moving away from 
a logarithmic function, however, a degree of swelling is presented that stabilizes asymptotically at an equilibrium value $\left(W t_{2}\right)$. Additionally, note that changes in the absolute value of swelling are observed between the two types of the curve (Fig. 2A and 2B), reaching lower degrees of swelling. These changes are due to irreversible transformations generated by possible breaks in the cross-linked bridges between PVA and PS caused by the hydration of the material.

Likewise, Fig. 2C and 2D shows the Swelling kinetic relations $(t / w)$ referring to the swelling data, where $w$ is the swelling at a given time $t$. In Fig. $2 \mathrm{C}$ it can be seen that the plotted function of the $t / w$ relationship has a behavior very close to linearity, characteristic that is verified with the correlation coefficient $\mathrm{R}^{2}$ which expresses a very high linear correlation for all hydrogels (see Table 2). This high correlation decreases slightly after the first swelling as observed in Fig. 2D, where it is observed that the correlation loses affinity, obtaining in some cases moderate correlations caused by the disruptions generated by hydration. The quantitative and qualitative assessments of the correlation coefficients can be examined in Table 2 .

The experimental swelling data were evaluated by the Schott's pseudo-secondorder swelling kinetics mathematical model to evaluate the swelling kinetics of the hydrogels, the model initially formulated by Equation 2:

$$
\frac{t}{w}=A+B t \quad \text { (Equation 2) }
$$

Indicating that:

$$
A=\frac{1}{k_{s} * W_{\infty}^{2}} \quad \text { (Equation 3) } \quad \mathrm{B}=\frac{1}{W_{\infty}} \quad \text { (Equation 4) }
$$

where $\mathrm{A}$ is the intercept with the vertical axis and $\mathrm{B}$ is the linear slope of the line $t / w$ obtained through the linear least-squares fit of the experimental data, see Equations 3 and 4. In this context, such constants allow us to determine the values of $K s$ and $W \infty$ can be rewritten the equation 2 as:

$$
\frac{t}{w}=\frac{1}{k_{S} * W_{\infty}^{2}}+\frac{t}{W_{\infty}} \quad \text { (Equation 5) }
$$

where $K s$ represents the swelling rate constant and $W \infty$ is the swelling in theoretical equilibrium. It is possible to verify that the experimental and theoretical values of first swelling are significantly close without greater variations, on the other hand, the model loses significance in the second swelling, see 
Table 2. For the case of the first swelling $W \infty$, some hydrogels (H1, H3, H4, H7, H8, and H9) are close to a higher value due to a asymptotical trend of increasing swelling of the hydrogel. On the other hand, other hydrogels (H2, H5, and H6) approach values lower than the experimental value; this trend is due to slight variations that oscillate around the equilibrium experimental swelling value, oscillations that were registered at the moment of mass reading. For the second swelling, an approximation of $W \infty$ to values greater than the experimental $W t_{2}$ is observed, an expected result given that all curves are constantly increasing swelling throughout the data collection record. In addition to that, greater differences are observed between the theoretical and experimental swellings, indicating a lower adjustment of the model for swelling after the initial one. The results obtained from the swelling curves adjusted to the Schott model can be seen in 


\section{Table 2.}
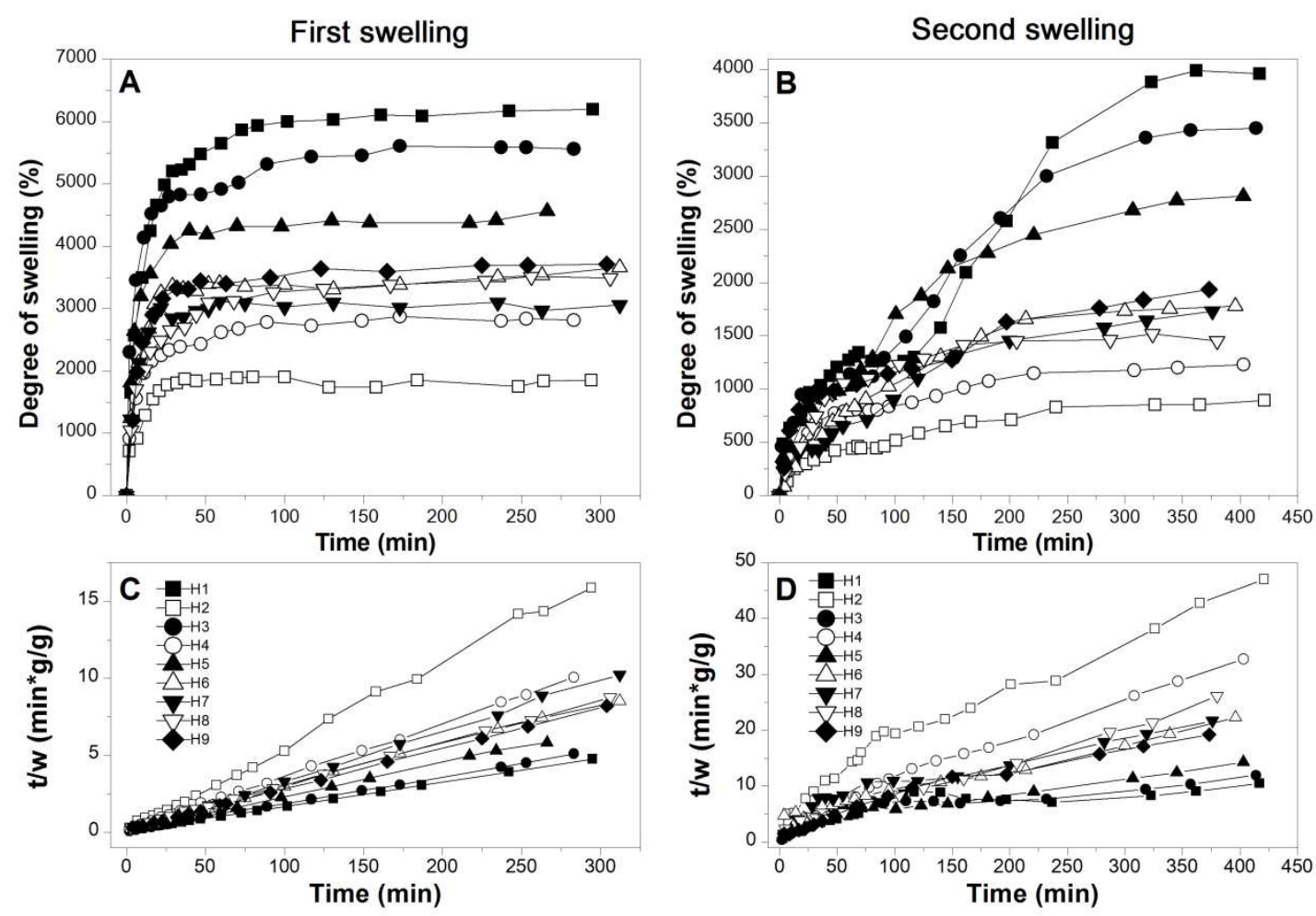

Fig. 2 Swelling kinetic curves and kinetic relations of hydrogels 
Table 2 Swelling kinetics and diffusion parameters of the hydrogel

\begin{tabular}{|c|c|c|c|c|c|c|c|c|c|c|}
\hline \multirow{2}{*}{$\begin{array}{c}N^{\circ} \\
\operatorname{Exp}\end{array}$} & \multicolumn{5}{|c|}{ First swelling } & \multicolumn{5}{|c|}{ Second swelling } \\
\hline & $W_{t}^{*}(\%)$ & $W_{\infty}(\%)$ & $K_{S}$ & $K_{I S}$ & $R^{2}$ & $W_{t}(\%)$ & $W_{\infty}(\%)$ & $K_{S}$ & $K_{I S}$ & $R^{2}$ \\
\hline H1 & 6196,4 & 6357,3 & $2,18 \mathrm{E}-03$ & $1,38 \mathrm{E}-01$ & 0,99991 & 3964,6 & 5299,4 & $1,01 \mathrm{E}-04$ & $5,34 \mathrm{E}-03$ & 0,62145 \\
\hline H2 & 1853,4 & 1833,2 & $3,19 \mathrm{E}-02$ & $5,86 \mathrm{E}-01$ & 0,99765 & 895,6 & 1031,1 & $1,26 \mathrm{E}-03$ & $1,30 \mathrm{E}-02$ & 0,97258 \\
\hline H4 & 2814,6 & 2890,2 & $6,04 \mathrm{E}-03$ & $1,75 \mathrm{E}-01$ & 0,99958 & 1230,7 & 1337,2 & $1,73 \mathrm{E}-03$ & $2,31 \mathrm{E}-02$ & 0,99219 \\
\hline H5 & 4561,1 & 4531,0 & 5,54E-03 & $2,51 \mathrm{E}-01$ & 0,99940 & 2812,7 & 3522,4 & 2,72E-04 & $9,59 \mathrm{E}-03$ & 0,93880 \\
\hline H6 & 3661,1 & 3615,3 & $5,50 \mathrm{E}-03$ & $1,99 \mathrm{E}-01$ & 0,99845 & 1778,3 & 2313,2 & 4,03E-04 & 9,31E-03 & 0,98972 \\
\hline H8 & 3518,1 & 3602,3 & $2,95 \mathrm{E}-03$ & $1,06 \mathrm{E}-01$ & 0,99971 & 1454,3 & 1647,7 & $1,70 \mathrm{E}-03$ & $2,80 \mathrm{E}-02$ & 0,99629 \\
\hline H9 & 3712,3 & 3777,9 & $4,51 \mathrm{E}-03$ & $1,70 \mathrm{E}-01$ & 0,99980 & 1938,3 & 2033,8 & $1,10 \mathrm{E}-03$ & 2,23E-02 & 0,96429 \\
\hline
\end{tabular}

$\boldsymbol{W}_{\boldsymbol{t}}$ : Experimental equilibrium swelling (Responses*), $\boldsymbol{W}_{\infty}$ : Equilibrium theoretical swelling, $\boldsymbol{K}_{\boldsymbol{S}}$ : Constant rate for swelling, $\boldsymbol{K}_{\boldsymbol{I S}}$ : Initial swelling constant, $\boldsymbol{R}^{2}$ : Correlation Coefficient.

Interpretation of correlation $\boldsymbol{R}^{2}$ : Very high correlation: __. High correlation: _. . Moderate correlation:

Fig. 3 shows the kinetic deswelling curves of the hydrogels, this test was carried out at a constant temperature of $40{ }^{\circ} \mathrm{C}$, simulating a drastic ambient temperature. Additionally, these tests were carried out sequentially after the swelling tests, which explains the initial values of the deswelling curves. On the other hand, as expected, the materials show reductions in their mass throughout the evaluated period until reaching an equilibrium mass, this deswelling curves it can be seen that the mass loss presents a partially sinusoidal behavior with an asymptotic end of the function (Fig. 3A and 3B). This partially sinusoidal behavior suggests the existence of a loss of critical mass which was evaluated by calculating the first derivative of the function of the deswelling curve (Fig. 3C and 3D). The composition of the different hydrogels analyzed determines the critical mass loss time, however, critical loss ranges occur in periods between 205 and 285 minutes for most of the hydrogels for the two deswelling cycles. It should be noted that the variation in drying temperature would vary the behavior of critical mass loss, displacing it positively or negatively in the exposure time. 
First deswelling
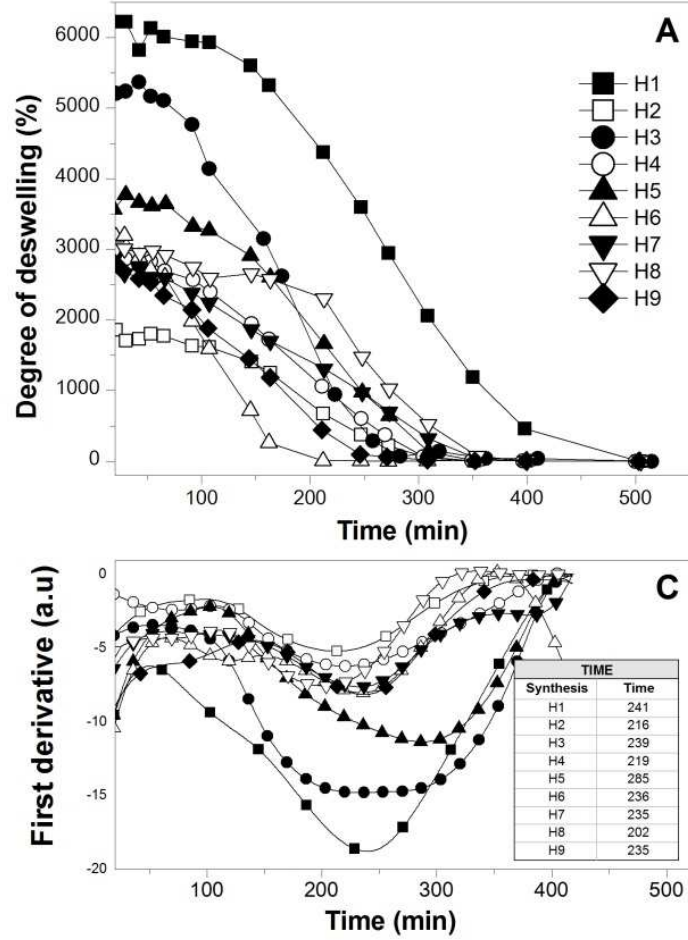

Second deswelling
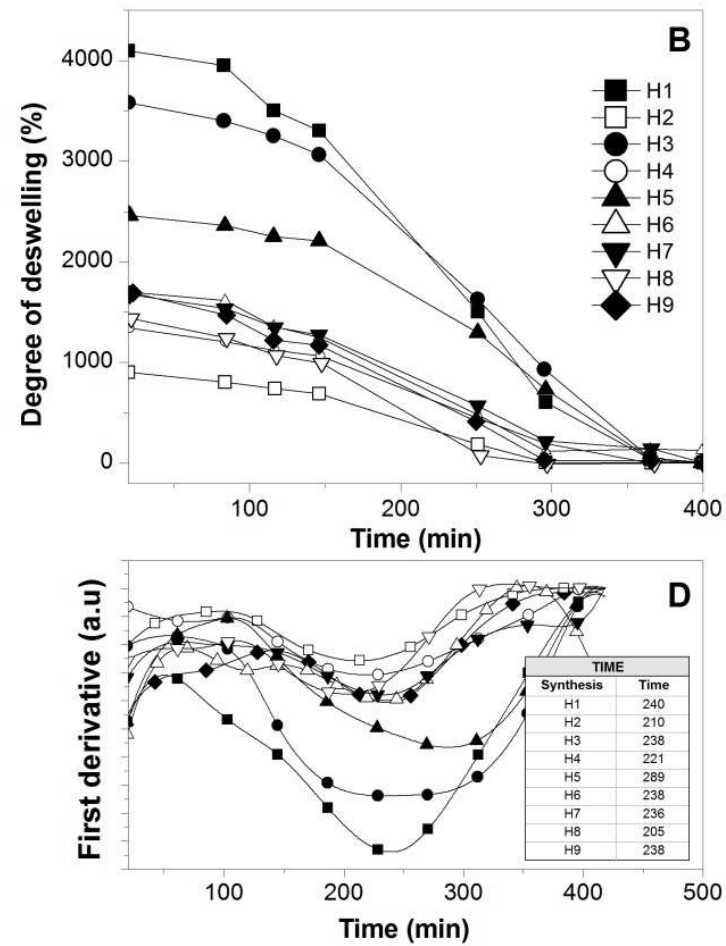

Fig. 3 Deswelling kinetic curves

\section{Statistical study}


Table 3 shows the experimental points and the experimental results of swelling used for the statistical study. Nine random runs were performed to reduce the errors of the systematic trends in the variables. The data obtained were evaluated by the analysis of variance (ANOVA) to adjust the response model by the least-squares method and to evaluate its adequate fit. The significance of the model was evaluated using the probability value ( $p$-value); at a 95\% confidence level, knowing that $p$-values lower than 0.065 indicates significant effects. The $p$-value coefficients greater than 0.065 were not significant for the model, so they were not considered in the regression model.

The statistical significance and the proposed adequacy of the developed equation, as well as the effects of the individual terms and their interactions, were analyzed by ANOVA method as summarized in Table 4. The fit of the model was evaluated using the probability value ( $p$-value), and the coefficients of determination $\left(\mathrm{R}^{2}\right.$ and $\mathrm{R}_{\mathrm{adj}}{ }^{2}$ ). Based on the parameters in Table 4, the cubic regression model with determined coefficients is given by Equation 6 . 
Table 3 Analysis of variance (ANOVA) for the polynomial model

\begin{tabular}{lcrrr}
\hline Sources & DF & Adj SS & Adj MS & p-value \\
\hline Regression & 6 & 14713095 & 2452182 & 0,014 \\
$\quad$ Lineal & 2 & 1492987 & 746493 & 0,044 \\
Quadratic & 2 & 1013440 & 506720 & 0,064 \\
$\quad$ PS*PVA & 1 & 965529 & 965529 & 0,034 \\
$\quad$ PS*MBAm & 1 & 762070 & 762070 & 0,043 \\
$\quad$ Special cubic & & & & \\
$\quad$ PS*PVA*MBAm & 1 & 486853 & 486853 & 0,065 \\
$\quad$ Full cubic & & & & \\
$\quad$ PS*PVA*(-) & 1 & 806540 & 806540 & 0,040 \\
\hline Error residual & 2 & 69495 & 34748 & \\
\hline Total & 8 & 14782590 & &
\end{tabular}

*DF: degree of freedom; Adj SS: Adjusted sums of squares; Adj MS: Adjusted mean squares; $F$-value; $\boldsymbol{p}$-value

$$
\begin{gathered}
Y=507 x_{1}+78545 x_{2}-201919 x_{3}-144310 x_{1} x_{2}+252769 x_{1} x_{3}+409246 x_{1} x_{2} x_{3}+ \\
65476 x_{1} x_{2}\left(x_{2}-x_{1}\right) \quad \text { (Equation 6) }
\end{gathered}
$$

Table 4 Regression coefficients

\begin{tabular}{ccrrr}
\hline Term & $\boldsymbol{\beta}_{\boldsymbol{x}}$ & \multicolumn{1}{c}{ Coef } & SE coef. & $\boldsymbol{p}$-value \\
\hline PS & $\beta_{1}$ & 507 & 438 & - \\
PVA & $\beta_{2}$ & 78545 & 13146 & - \\
MBAm & $\beta_{3}$ & -201919 & 46631 & - \\
PS *PVA & $\beta_{12}$ & -144310 & 27376 & 0,034 \\
PS * MBAm & $B_{13}$ & 252769 & 53974 & 0,043 \\
PS * PVA* MBAm & $B_{123}$ & 409246 & 109332 & 0,065 \\
PS * PVA*(-) & $B_{4}$ & 65476 & 13590 & 0,040 \\
\hline
\end{tabular}

The data of the model ( 
Table 3) show that the $p$-values, less than or equal to 0.065 , indicates that the predictive cubic model was significant concerning the experimental data selected from the swelling kinetic curves. The significance and adequacy of the models were also verified by determining the coefficients of $R^{2}$ and $R_{a d j}^{2}\left(R^{2}=99,53 \%\right.$ and $R_{a d j}^{2}=$ $98,12 \%)$, showing a high degree of prediction when compared with experimental data. These values suggest that the model fits $\sim 98 \%$ of the variability of the experimental data on average and a percentage less than $2 \%$ of the variation in the content of PS, PVA, and MBAm that could not be adjusted by the developed model. These values $\left(R^{2}\right.$ and $R_{a d j}{ }^{2}$ ) therefore show a high significance of the model and a correlation between the predicted and observed values based on the proposed relevance analysis, this concordance can be observed in parallel in the results obtained in the residual graphs (Fig. 4A).

The Anderson Darling statistical test was developed, which generated a $p$-value of $\sim 0.064$, a value slightly above the usual value of the significance level; this result suggests that the residues describe a predominantly normal distribution, see Fig. 4A3. On the other hand, the independence of the results was established through the DurbinWatson test, the data and results related to this test can be seen in Table 5 and Equation 7. In this context, the Durbin-Watson result generated in the test $(\sim 2,58)$ is in an accepted band that indicates that the data are approximately independent or without appreciable correlation.

The proposed model allowed us to understand and corroborate various aspects of the effects between the variables studied; initially, it allows us to determine two large regions of statistical relevance, which denote positive and negative statistical significance, see Fig. 4B. This is because, with incorporations greater than $\sim 23 \mathrm{wt} \%$ of the MBAm crosslinking agent a negative effect is exerted on the response, these significant amounts of MBAm incorporated in the hydrogel drastically change the structure and its behavior about swelling. Effects associated with excess crosslinking produce a decrease in the elongation capacity of the polymeric chains, greater rigidity of the material and of the cavities that stock the absorbable liquid, producing in the hydrogel reduced or irregular liquid retention and swelling capacities. The said phenomenon of changes in the swelling capacity related to the increase in crosslinking agent was already reported in previous studies that described similar behaviors [17,2123]. 
On the other hand, the interactive effects of the variables studied on the response (Wt) were analyzed by drawing level curves in the ternary diagram as a function of the three variables (PS, PVA, and MBAm), see Fig. 4B. When observing the level curves, close to the PS-PVA axis, it can be observed that reciprocity occurs in which the response increases with the gradual incorporation of the PVA component.

Table 5 Durbin-Watson test data

\begin{tabular}{|c|c|c|c|c|c|}
\hline \multirow{2}{*}{ Sample } & \multicolumn{2}{|c|}{ Swelling } & \multirow[b]{2}{*}{ Residue } & \multicolumn{2}{|c|}{ Durbin-Watson Test } \\
\hline & $\boldsymbol{W}^{*}$ & $\boldsymbol{W}_{\infty S M^{* *}}$ & & $e_{i}^{2}$ & $\left(e_{i}-e_{i-1}\right)^{2}$ \\
\hline H1 & 6196,36 & 6202,19 & $-5,83$ & 33,93 & --- \\
\hline $\mathrm{H} 2$ & 1853,37 & 1859,39 & $-6,02$ & 36,28 & 0,04 \\
\hline H3 & 5562,04 & 5527,99 & 34,05 & 1159,31 & 1605,77 \\
\hline $\mathrm{H} 4$ & 2814,64 & 2791,54 & 23,10 & 533,61 & 119,87 \\
\hline H5 & 4561,08 & 4537,88 & 23,20 & 538,09 & 0,01 \\
\hline H6 & 3661,08 & 3797,61 & $-136,53$ & 18639,40 & 25511,42 \\
\hline $\mathrm{H} 7$ & 3058,57 & 3024,64 & 33,93 & 1151,52 & 29056,68 \\
\hline $\mathrm{H} 8$ & 3518,11 & 3654,58 & $-136,47$ & 18623,88 & 29037,29 \\
\hline H9 & 3712,33 & 3542,69 & 169,64 & 28779,16 & 93705,50 \\
\hline \multirow{2}{*}{\multicolumn{4}{|c|}{$\begin{array}{l}* W_{t} \text { : Equilibrium swelling. } \\
* * W_{\infty} S M \text { : Equilibrium theoretical swelling of statistical model. }\end{array}$}} & $\sum_{i=1}^{n} e_{i}^{2}$ & $\sum_{1=2}^{n}\left(e_{i}-e_{i-1}\right)^{2}$ \\
\hline & & & & 69495,18 & 179036,58 \\
\hline
\end{tabular}

$$
D W=\frac{\sum_{1=2}^{n}\left(e_{i}-e_{i-1}\right)^{2}}{\sum_{i=1}^{n} e_{i}^{2}}=\sim 2,58 \quad(\text { Equation 7) }
$$

A1

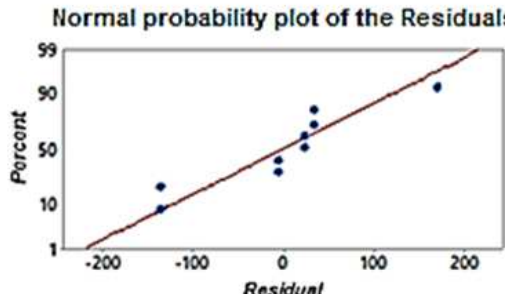

A2

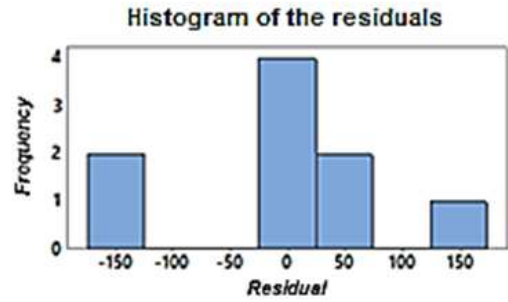

A3

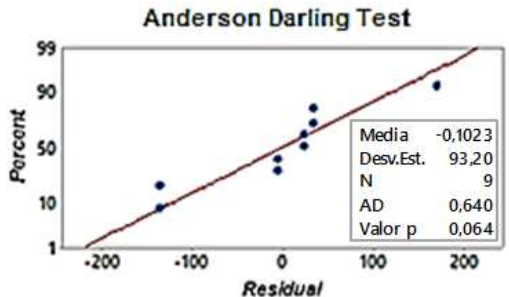

B

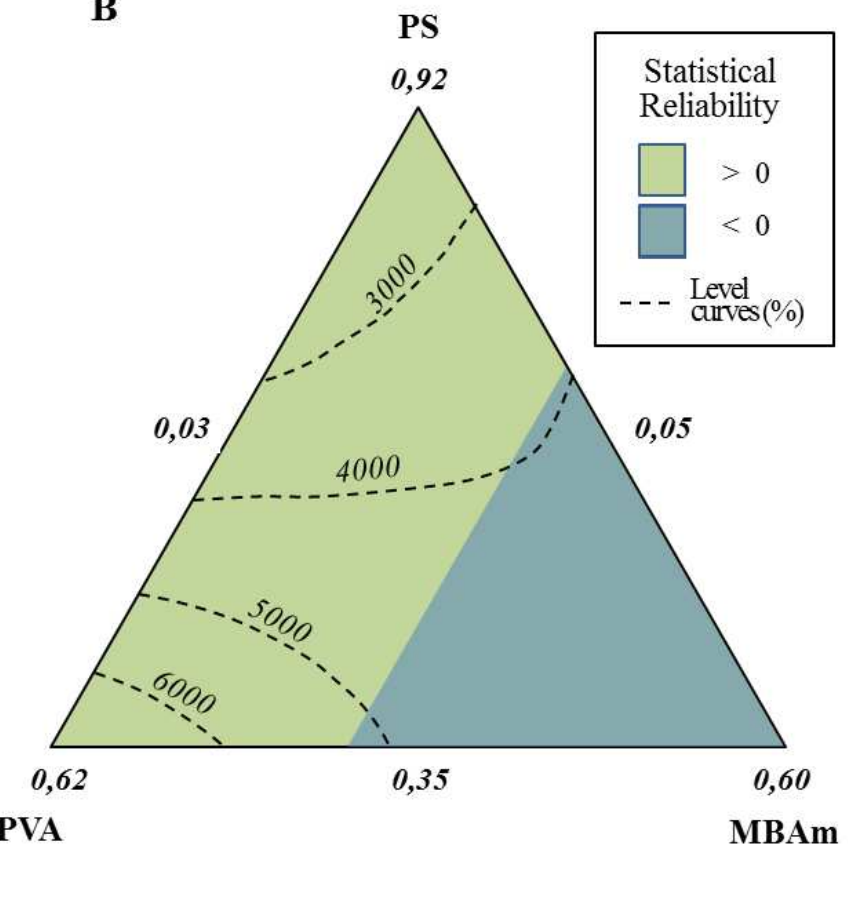

Fig. 4 Statistical graphics: A) Residual plots. B) Contour plots for $\mathrm{Wt}_{1}$ content vs. PS, PVA and MBAm 
Fig. 5 shows the general results of the experimental $(W t)$ and theoretical $(W \infty)$ swellings in equilibrium referring to the Schott and statistical models, data extracted from Tables 2 and 5. In these results, it is possible to observe that the data are expressively close to the experimental data for each type of hydrogel. The comparison of the absolute values of the models together with the experimental results allows to verify the proximity in the results by hydrogel, allowing the indirect validation of the studied models, since, although the models have different foundations, they provide results and responses associated with the theoretical swelling in equilibrium of the hydrogels studied.

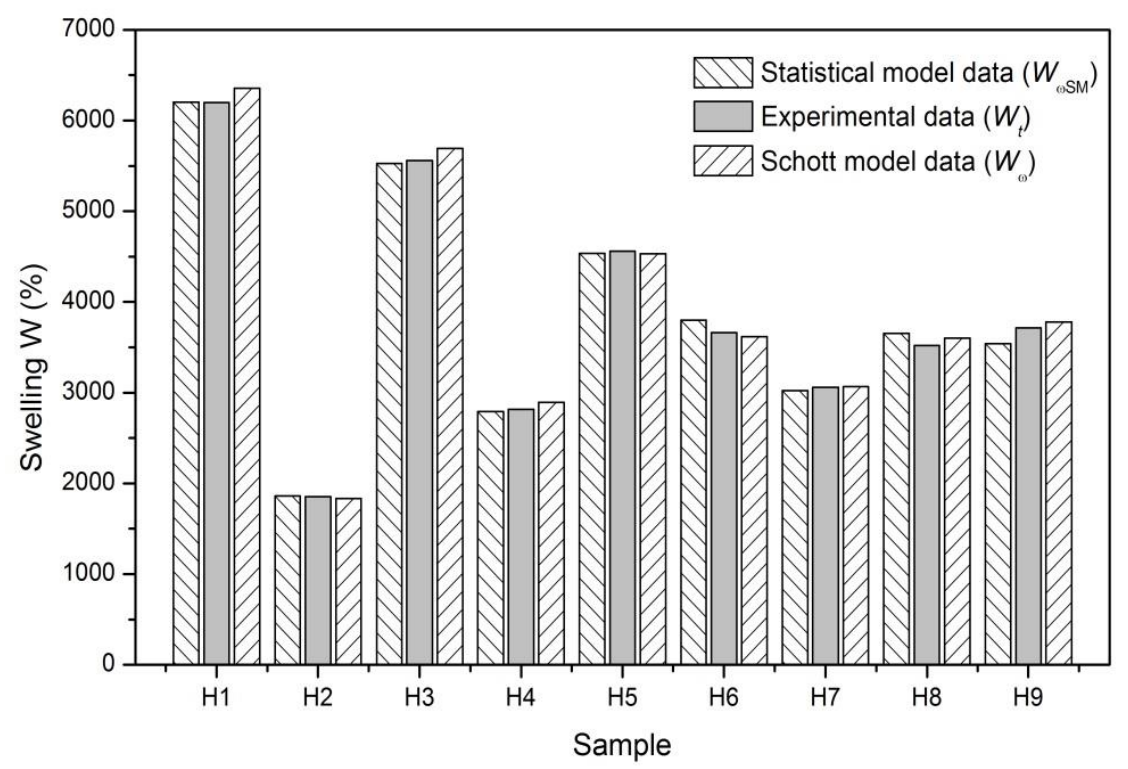

Fig. 5 Comparison of equilibrium swellings

\section{FTIR analysis}

The main raw materials used in the present work were characterized by FTIR, to verify the transformations generated after the synthesis. In this context, Fig. 6 shows the FTIR spectra of PVA, PS, and MBAm, in the case of PVA spectrum the typical bands were identified of the presence by hydroxyl groups $\mathrm{O}-\mathrm{H}$ at $\sim 3301 \mathrm{~cm}^{-1}$, stretching $\mathrm{C}-\mathrm{H}$ from alkyl groups at $\sim 2925 \mathrm{~cm}^{-1}$, bands of bonds deformations of $\mathrm{C}=\mathrm{O}$ and $\mathrm{C}-\mathrm{O}$ at $\sim 1733-1716 \mathrm{~cm}^{-1}$, bending vibration mode of $-\mathrm{CH}_{2}$ group at $\sim 1436 \mathrm{~cm}^{-1}$, stretching vibration of $\mathrm{C}-\mathrm{O}-\mathrm{C}$ and $\mathrm{C}-\mathrm{OH}$ at $\sim 1139$ and $\sim 1091 \mathrm{~cm}^{-1}$, respectively, band of ester stretch and $\mathrm{C}-\mathrm{O}$ bond at $1025 \mathrm{~cm}^{-1}$ and deformation outside the $\mathrm{O}-\mathrm{H}$ band plan at $\sim 947$ $\mathrm{cm}^{-1}[1,3,8,24-27]$. 
FTIR spectra of PS (Fig. 6) shows hydroxyl groups O-H at $\sim 3315 \mathrm{~cm}^{-1}$, stretching $\mathrm{C}-\mathrm{H}$ from alkyl groups at $\sim 2929$ and $\sim 1150 \mathrm{~cm}^{-1}$, the band of deformation from $\mathrm{CH}_{2}$ and $-\mathrm{COO}$ at $\sim 1417$, vibrations of the $\mathrm{C}-\mathrm{O}-\mathrm{C}$ and glycosidic bond can present different modes of vibrations and bending conformations at $\sim 1155$ and $\sim 927 \mathrm{~cm}^{-1}$, the band of C-C stretch at $\sim 769 \mathrm{~cm}^{-1}$, and the bands of stretching pyranose ring at $\sim 1004$ and $\sim 574 \mathrm{~cm}^{-1}$ [19-20,25,28-29]. On the other hand, the FTIR spectra of MBAm shows bands of deformations N-H bond at 3309, 3037, and $1541 \mathrm{~cm}^{-1}$, bands of deformations $\mathrm{N}-\mathrm{H}$ bond at 1307 and $955 \mathrm{~cm}^{-1}$, several stretching of $\mathrm{CH}_{2}$ at 3107, 3070, 2960, 1412 , 1384, 1436, $989 \mathrm{~cm}^{-1}$, two stretching of C-H at 2854 and $1072 \mathrm{~cm}^{-1}$, deformations at 1657, 1625, 967 and $479 \mathrm{~cm}^{-1}$ caused by $\mathrm{C}=\mathrm{O}, \mathrm{C}=\mathrm{C}$ and $\mathrm{C}-\mathrm{C}$ bond, and stretching at 619 $\mathrm{cm}^{-1}$ caused by $\mathrm{O}=\mathrm{C}-\mathrm{N}$ group [30].

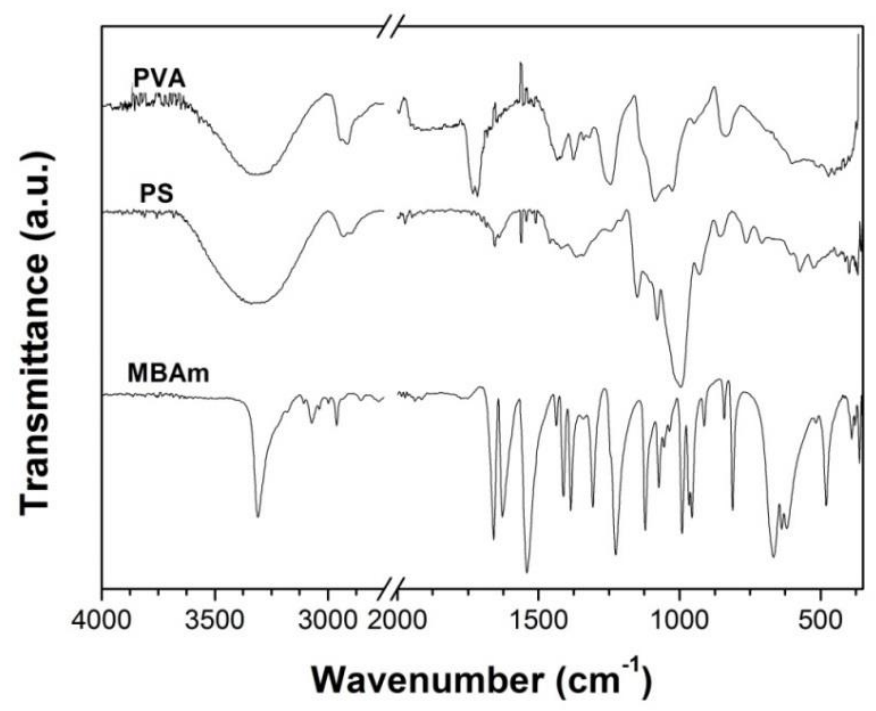

Fig. 6 FTIR spectra of raw materials

Fig. 7 shows the spectra of the hydrogels produced, where can be observed the presence of hydroxyl groups (O-H bond) of PVA and PS at $3301 \mathrm{~cm}^{-1}$ [24-25, 31-35], $\mathrm{C}-\mathrm{H}$ stretching peak of the PS at $2930 \mathrm{~cm}^{-1}$ [25,31,34-36], the band at $1670 \mathrm{~cm}^{-1}$ ascribed to the presence of residual acetate groups present in PVA, the observed band also implies for N-H stretching due to MBAm. Similarly, strong amide bands between $1590-1490 \mathrm{~cm}^{-1}$ may also be assigned to $\mathrm{C}=\mathrm{O}$ and $\mathrm{C}=\mathrm{C}$ stretching vibration from effective crosslinking of MBAm.

In addition, the band represents the $\mathrm{CH}_{2} \mathrm{OH}$ stretching vibration of the PS (C-O stretching) $1240 \mathrm{~cm}^{-1}$ [31,34-35], band near $1152 \mathrm{~cm}^{-1}$ suggests the robust inter- and intramolecular interactions of hydroxyl groups [25,32-33], the stretch near $1085 \mathrm{~cm}^{-1}$ 
arises as a result of crosslinking reaction among PVA/PS chains and MBAm $(\mathrm{C}-\mathrm{O}-\mathrm{C}$ group). This also suggests the formation of ether linkages and acetyl rings. The strong band at $1020 \mathrm{~cm}^{-1}$ represents the ether linkages and acetal ring as a result of the reaction between the MBAm and $\mathrm{OH}$ group of the PS and PVA. Likewise, the crosslinking generated by MBAm is reflected in several bands of different intensities between the 1200 and $1000 \mathrm{~cm}^{-1}$ range through the formation of the C-O-C group. At last, the $\mathrm{CH}_{2}$ wagging band at $850 \mathrm{~cm}^{-1}$ and the small bands 580 and $520 \mathrm{~cm}^{-1}$ appear due to the pyranose ring present in PS. 
Table 6 summarizes the characteristics of the bands identified in the FTIR spectra of the hydrogels. The generation of new characteristics in the spectra of the hydrogels compared with the raw materials, as well as the types of bands generated in the products studied proposed suggests that the crosslinking was carried out, confirming that the proposed synthesis methodology was effective concerning to integration of new hydrogels.

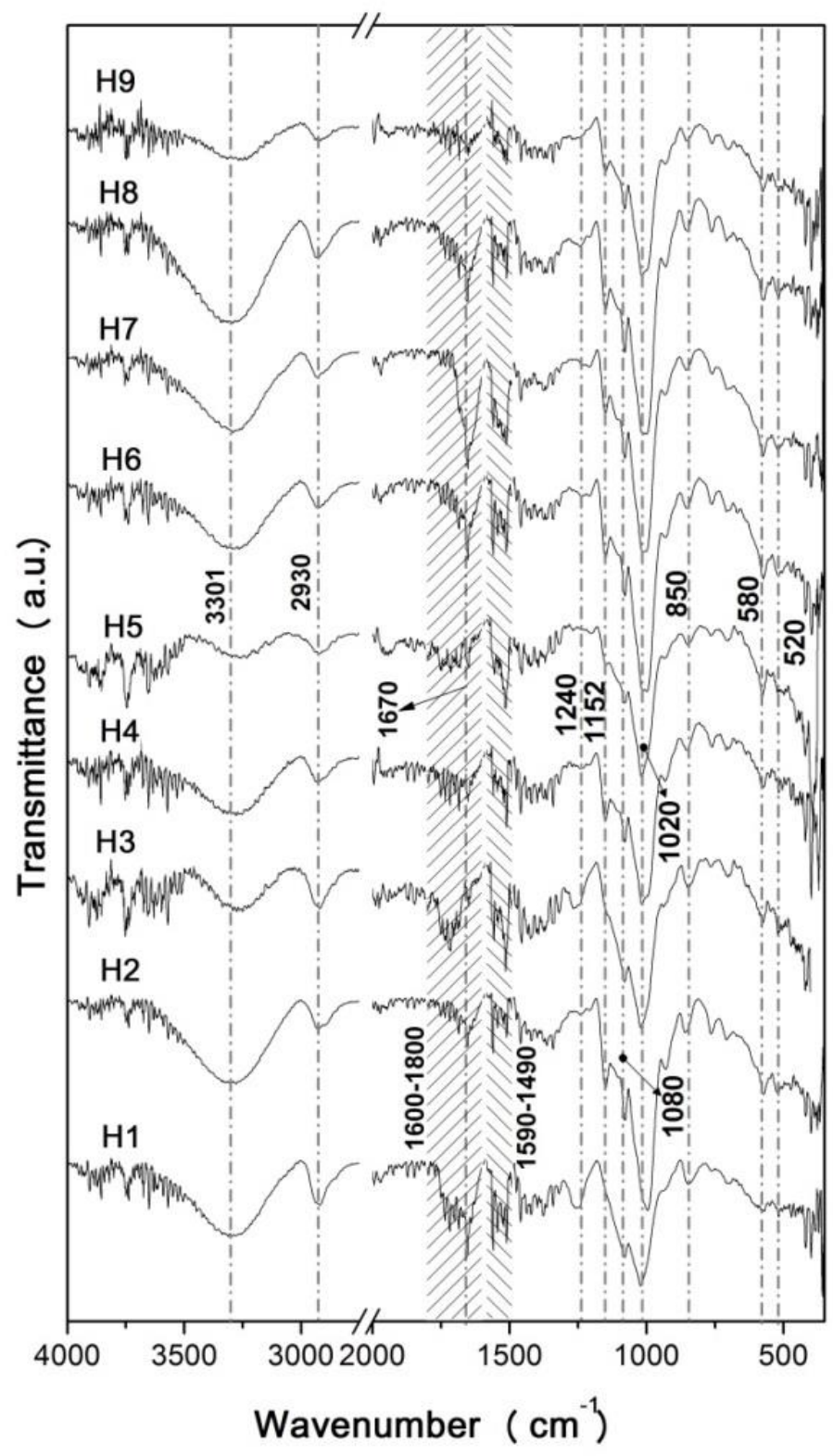

Fig. 7 Hydrogel FTIR spectra 
Table 6 FTIR Bands Summary

\begin{tabular}{|c|c|c|}
\hline Bands description & $\begin{array}{c}\text { Wavenumber } \\
\left(\mathbf{c m}^{-1}\right)\end{array}$ & Reference \\
\hline Presence of hydroxyl groups (O-H) group of PVA and PS. & 3301 & {$[24-25,31-35$} \\
\hline C-H stretching peak of the PS. & 2930 & {$[25,31,34-36]$} \\
\hline The band represents the $\mathrm{CH}_{2} \mathrm{OH}$ stretching vibration of the PS. (CO stretching) & 1240 & [31,34-35] \\
\hline $\begin{array}{l}\text { Band at } 1020 \mathrm{~cm}^{-1} \text { represents the ether linkages and acetal ring as a result of the } \\
\text { reaction between the MBAm and } \mathrm{OH} \text { group of the polymer. }\end{array}$ & 1020 & {$[31,37]$} \\
\hline $\begin{array}{l}\text { The band near } 1152 \mathrm{~cm}^{-1} \text { appeared as a result of intra- and intermolecular } \\
\text { interactions of hydroxyl groups in PVA and PS. }\end{array}$ & 1152 & {$[25,32-33]$} \\
\hline $\begin{array}{l}\text { A band at } 1085 \mathrm{~cm}^{-1} \text { is due to the crosslinking reaction among PVA/PS chains } \\
\text { and MBAm (C-O-C group). This suggests the formation of ether linkages and } \\
\text { acetyl rings. }\end{array}$ & 1085 & {$[25,32-34]$} \\
\hline $\begin{array}{l}\text { The observed band also implies NH stretching due to MBAm. Similarly, a } \\
\text { strong amide band between } 1590-1490 \mathrm{~cm}^{-1} \text { may also be assigned to the } \mathrm{C}=\mathrm{O} \\
\text { stretching vibration of MBAm and other groups. }\end{array}$ & 1550 & [36] \\
\hline Presence of residual acetate groups present in PVA. & 1670 & {$[33,37]$} \\
\hline $\begin{array}{l}\text { The peaks between } 1600 \text { and } 1800 \mathrm{~cm}^{-1} \text { signify the presence of both } \mathrm{C}=\mathrm{O} \text { and } \\
\mathrm{C}=\mathrm{C} \text { bonds. }\end{array}$ & $1600-1800$ & {$[24,38]$} \\
\hline $\begin{array}{l}\text { Several bands attributed to the carbonyl group (at } 1716-1718 \mathrm{~cm}^{-1} \text { ) were } \\
\text { observed in FTIR spectra. }\end{array}$ & $1600-1800$ & {$[35]$} \\
\hline $\mathrm{CH}_{2}$ wagging band at $854 \mathrm{~cm}^{-1}$. & 850 & [35] \\
\hline $\begin{array}{l}\text { The Bands in the range of } 900-500 \mathrm{~cm}^{-1} \text { appear due to the pyranose ring } \\
\text { present in PS. }\end{array}$ & 580,520 & {$[25,33]$} \\
\hline
\end{tabular}

\section{Thermal analysis}

The TGA thermograms can be observed in Fig. 8A, in these curves, it can be seen that each sample presents several mass losses that can be confirmed and defined with their respective first derivatives, see Fig. $8 \mathrm{~B}$ and 
Table 7. In this way, four main events were identified related to critical mass losses that are associated with the decomposition of the PVA side groups and the chain itself ( 208 and $\sim 413{ }^{\circ} \mathrm{C}$ ), on the other hand, critical mass losses by decomposition of starch and their respective amylose and amylopectin fractions were identified at temperatures of $\sim 212$ and $\sim 370{ }^{\circ} \mathrm{C}$, respectively. It should be noted that these events were not fully evidenced in all the TGA tests of the studied samples (see Fig. 8B and Table 7), this possibly due to the differences between compositions of the samples and due to the washes carried out that could remove fractions in different proportions that did not react during the synthesis.
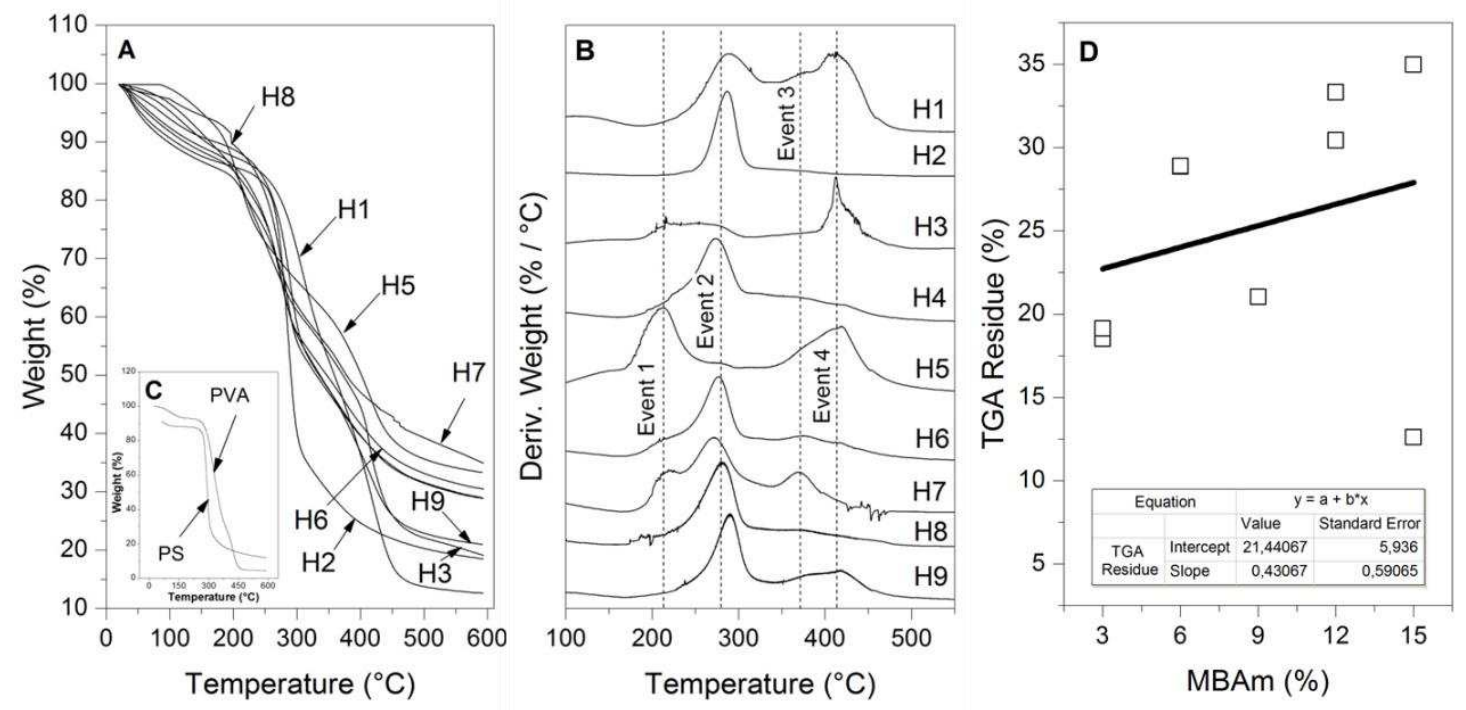

Fig. 8 Thermal results. A) TGA curves of hydrogels, B) Derivative weight, C) TGA curves of pure components, and D) DSC Thermograms of hydrogels

In the same way, Fig. 8A allows appreciating the percentage residues of the samples generated at the end of the thermal treatment of the TGA, these residues were examined finding an apparent relationship between the percentage of residue and the amount of MBAm incorporated in the synthesis. In this context, the percentage residuals generated in the TGA tests were plotted as a function of the amount of MBAm incorporated at the time of synthesis and treated by linear adjustment, see Fig. 8D. These results suggest a possible relationship between the amount of crosslinker and the thermal stability of the hydrogels; similar behavior was reported in other cross-linked polymeric works [39-44]. Increasing thermal stability in the function of the incorporation of MBAm is another evidence, together with the FTIR technique, for 
successful cross-linking of hydrogels. Also suggests that the crosslinker coupled to other structures such as PVA and PS can induce an improvement of thermal stability when compared to the performance of pure PVA and PS, see Fig. 8C. 
Table 7 Thermal events of TGA and DSC tests

\begin{tabular}{|c|c|c|c|c|c|c|}
\hline \multirow[b]{2}{*}{ Sample } & \multicolumn{4}{|c|}{ Thermal events in TGA test (loss of mass) } & \multirow{2}{*}{$\begin{array}{l}\text { TGA Final } \\
\text { residue } \\
(\%)\end{array}$} & \multirow[b]{2}{*}{$\begin{array}{l}\mathbf{T m} \\
\left({ }^{\circ} \mathbf{C}\right)\end{array}$} \\
\hline & $\begin{array}{c}\text { Event } 1 \\
\left(\sim 212^{\circ} \mathrm{C}\right)\end{array}$ & $\begin{array}{c}\text { Event } 2 \\
\left(\sim 280^{\circ} \mathrm{C}\right)\end{array}$ & $\begin{array}{c}\text { Event } 3 \\
\left(\sim 370^{\circ} \mathrm{C}\right)\end{array}$ & $\begin{array}{c}\text { Event } 4 \\
\left(\sim 413^{\circ} \mathrm{C}\right)\end{array}$ & & \\
\hline H1 & Not visible & Visible & Visible & Visible & $\sim 12,62$ & $\sim 126,17$ \\
\hline $\mathrm{H} 2$ & Not visible & Visible & Not visible & Not visible & $\sim 18,54$ & $\sim 248,17$ \\
\hline $\mathrm{H} 3$ & Visible & Visible & Not visible & Visible & $\sim 19,14$ & $\sim 197,17$ \\
\hline $\mathrm{H} 4$ & Not visible & Visible & Visible & Visible & $\sim 28,86$ & $\sim 203,17$ \\
\hline H5 & Visible & Visible & Visible & Visible & $\sim 33,33$ & $\sim 219,17$ \\
\hline H6 & Visible & Visible & Visible & Visible & $\sim 30,44$ & $\sim 208,17$ \\
\hline $\mathrm{H} 7$ & Visible & Visible & Visible & Not visible & $\sim 34,98$ & $\sim 216,17$ \\
\hline $\mathrm{H} 8$ & Not visible & Visible & Visible & Not visible & $\sim 28,91$ & $\sim 245,17$ \\
\hline H9 & Not visible & Visible & Visible & Visible & $\sim 21,03$ & $\sim 224,17$ \\
\hline
\end{tabular}

Fig. 9A shows the DSC thermograms of the hydrogels referring to the second heating, in these thermograms events were identified in the form of endothermic peaks associated with the melting temperature, which manifest themselves at different temperatures, see Table 7 . This behavior indicates the alteration in crystallinity that may be due to the change in the degree of crosslinking in the hydrogel, which implies a change as a function of the amount of crosslinking incorporated during the synthesis. These observed changes in crystallinity support previous studies where agents incorporated into PVA affect observable transitions in DSC tests, generating transition temperature shifts as in the case of melting point [10,45-49].
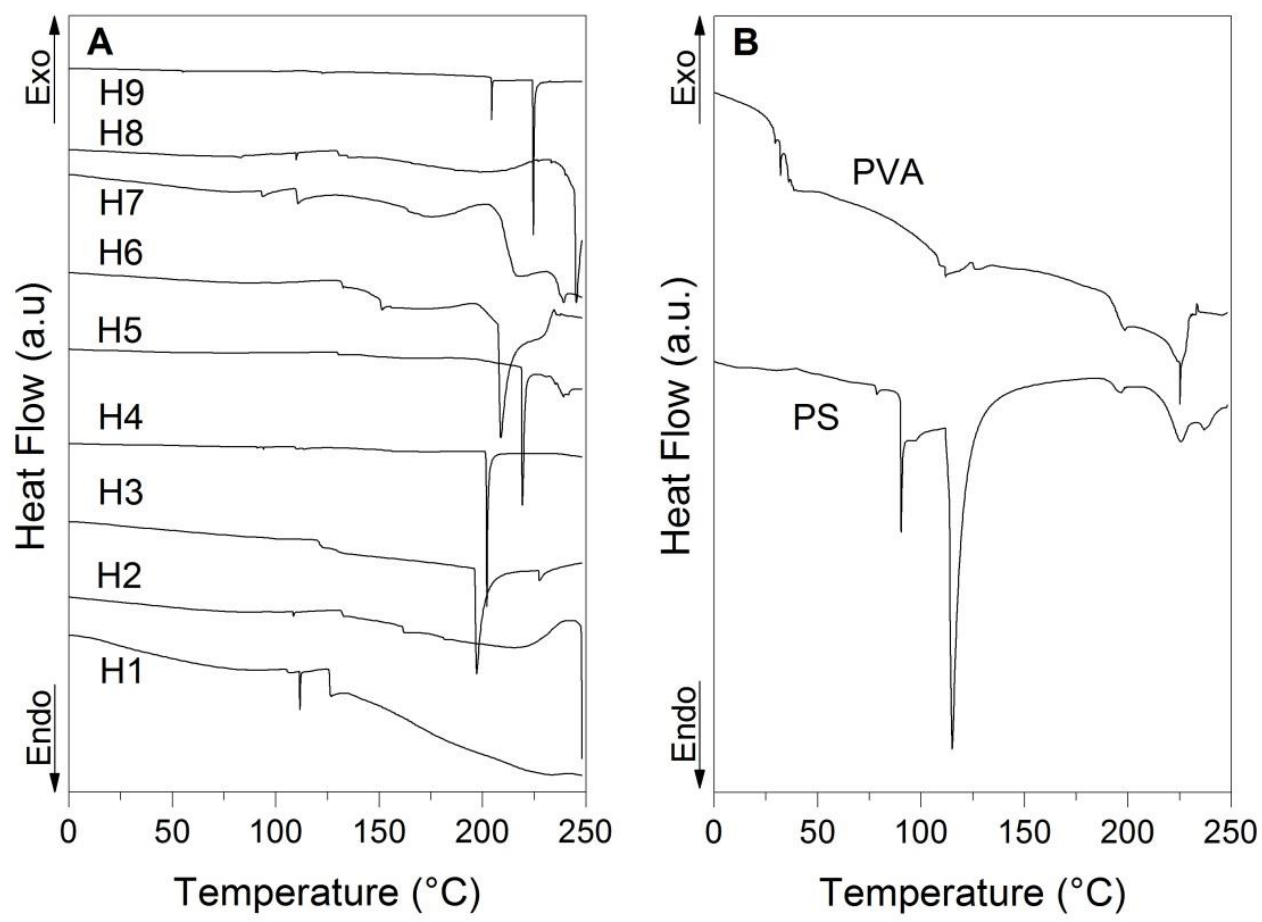

Fig. 9 DSC Thermograms: A) DSC curves of hydrogels and B) PVA and PS. 


\section{SEM analysis}

Fig. 10-12 show the SEM micrographs of the hydrogels named H2, H5, and H9, respectively, these micrographs were selected based on their swelling potential (high, medium, and low). In general, all the presented micrographs of the hydrogels show morphological characteristics of a composite material wherein the polymeric matrix constituted by PVA is visualized, integrating dispersed phases constituted by aggregate PS.

In most micrographs, the PVA polymer phase is characterized by predominantly smooth surfaces and the dispersed phase (PS) is characterized by having curved or flat granules with variable roughness and have spherical, polygonal, and oval shapes, morphological characteristics previously recorded in the literature [13,50]. Additionally, the polydispersity of the dispersed phase within the polymeric matrix is observed to be homogeneous with some agglomerations possibly produced in the drying and washing processes, however, the aggregates are present throughout the matrix.

Looking at it the other way, it can be seen in Fig. 10-12 various pores represented as black irregular dots or dark irregular cavities scattered in the matrix, additionally, an increase in the presence of pores is observed with the decrease of the PVA content and with the increase of the PS. Likewise, the presence of greater amounts of porosity is observed in the spaces of the agglomerated PS aggregate particles.

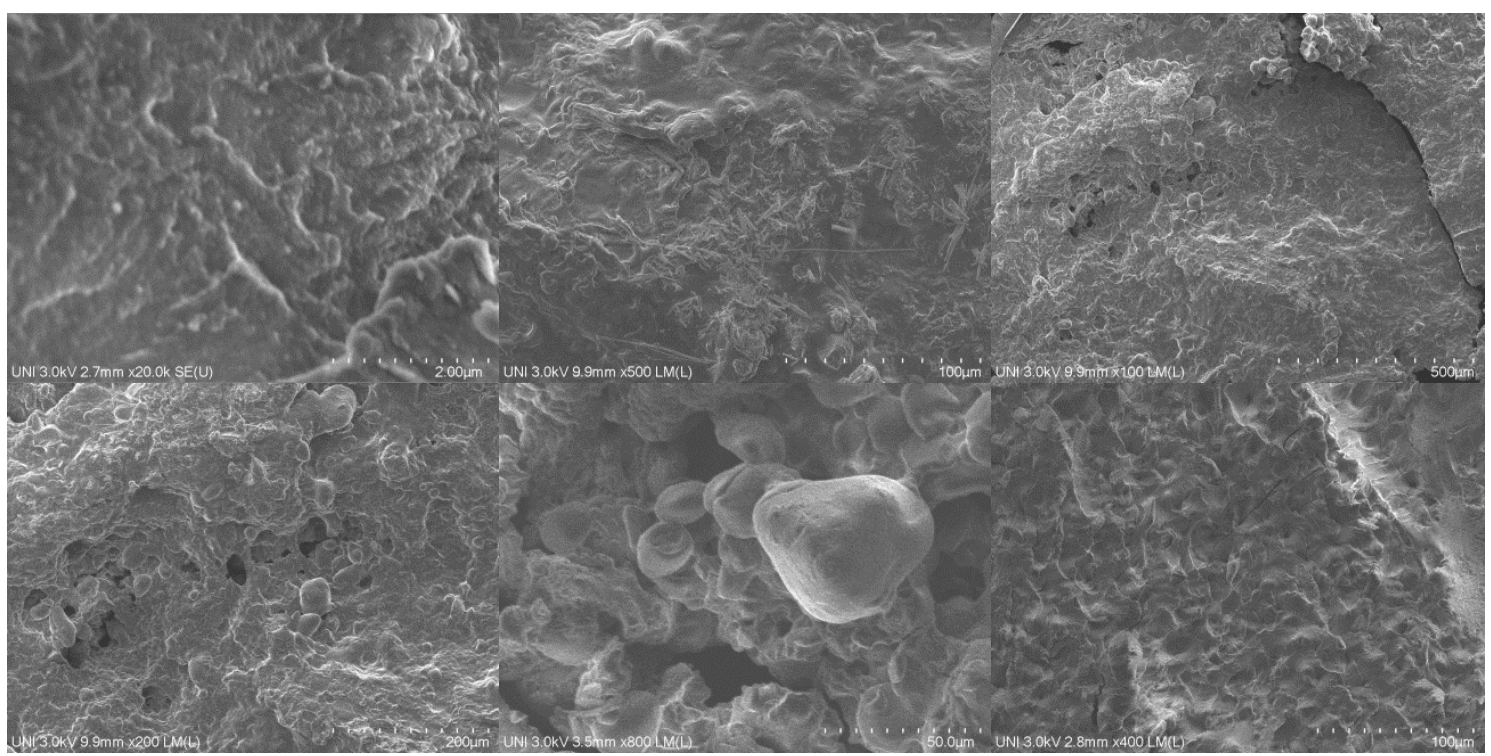

Fig. 10 SEM micrographs of hydrogels H2 


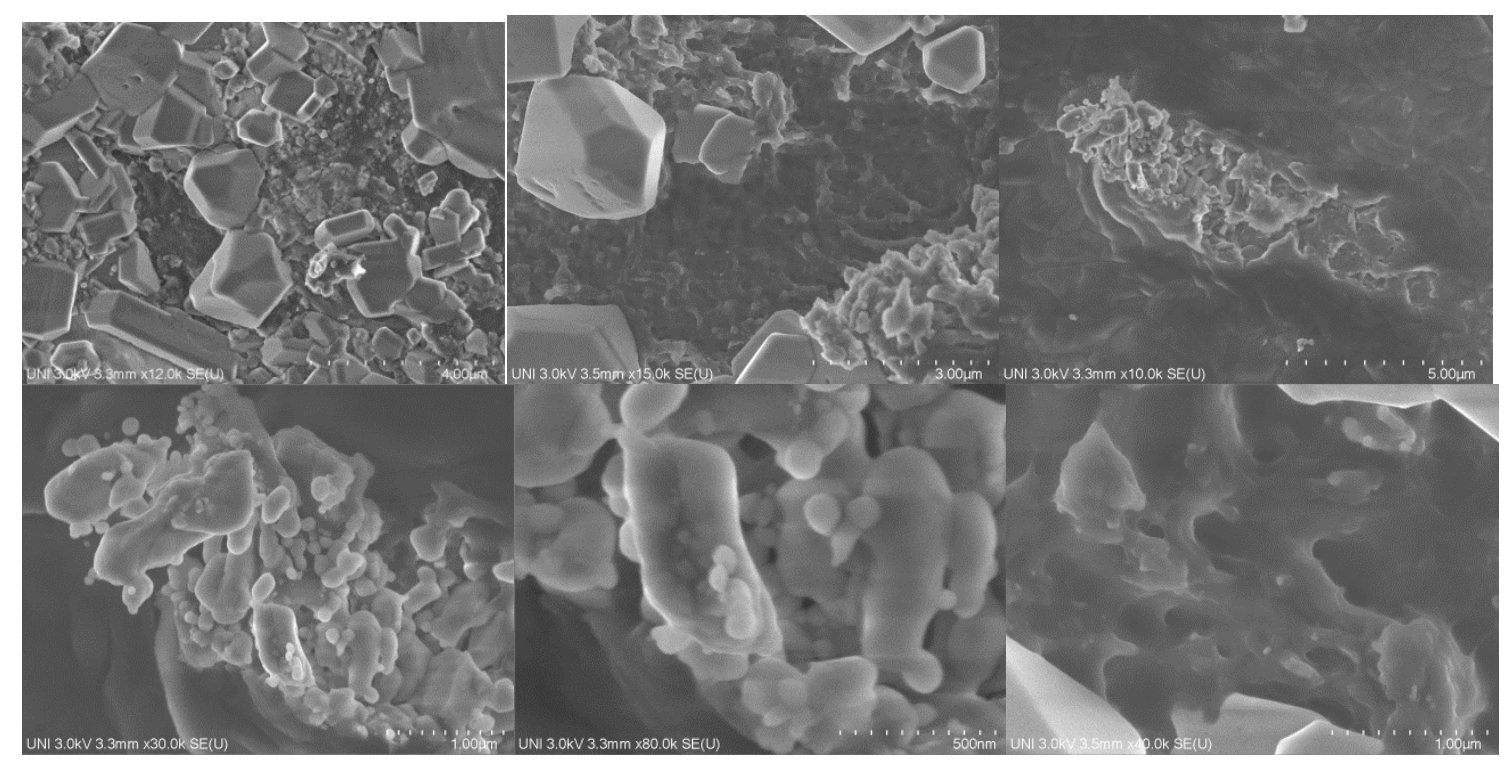

Fig. 11 SEM micrographs of hydrogels H5

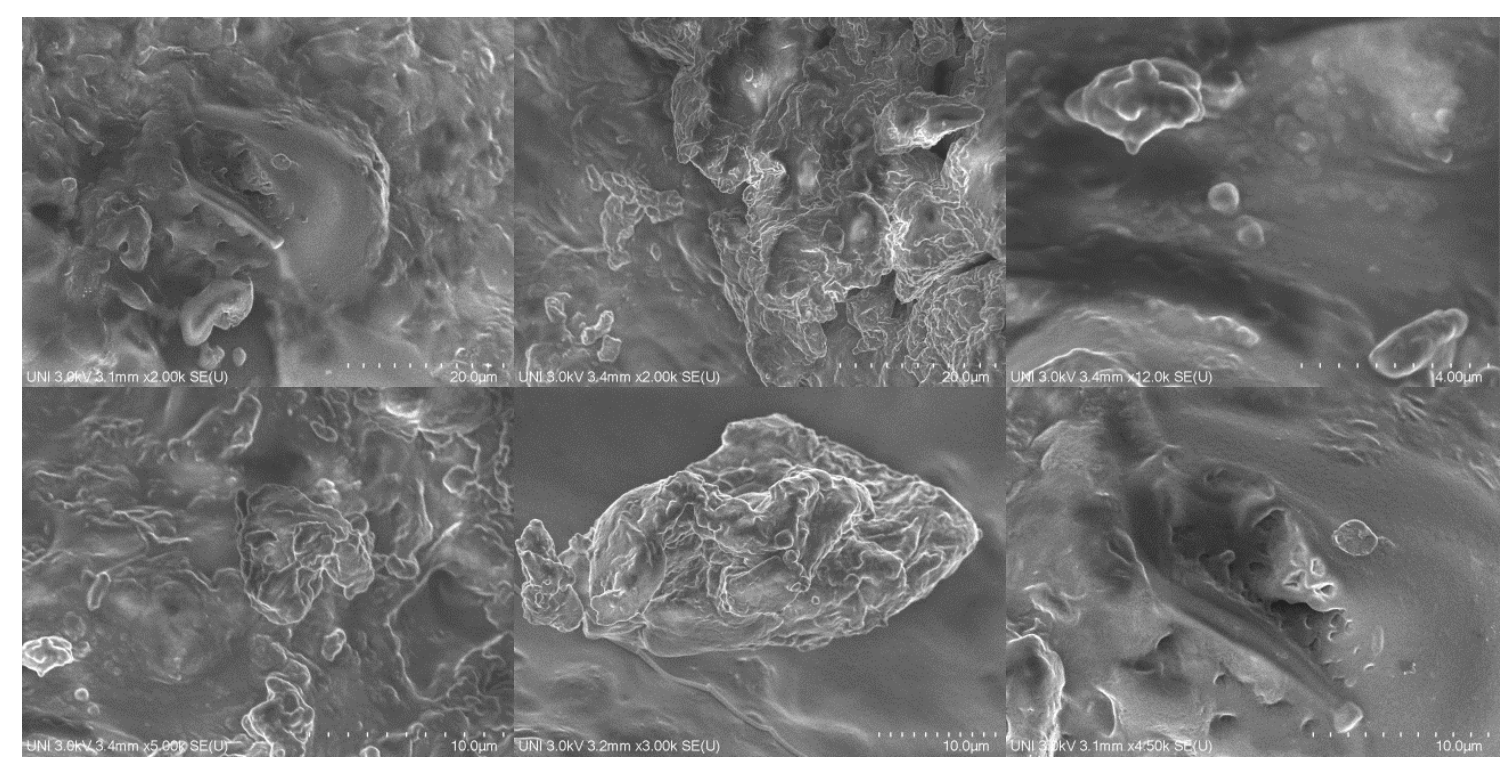

Fig. 12 SEM micrographs of hydrogels H9

\section{Conclusions}

Different hydrogels were synthesized by free-radical polymerization in an aqueous medium based on potato starch (PS), poly(vinyl alcohol) (PVA), and N,N'Methylenebisacrylamide (MBAm). Thermal and spectroscopic characterizations made it possible to determine the crosslinking of the raw materials, showing the viability of the synthesis and consequently the obtaining of hydrogels.

The results of swelling and deswelling of different hydrogels demonstrate that the synthesized material suffering irreversible disruptions and transitions after the first swelling and deswelling decreasing the degree of swelling. In addition, Schott's pseudo- 
second order swelling kinetics model fits the experimental results, however, the model loses affinity with the second swelling, generating a loss of relative precision, which must be considered when performing this type of cyclical test.

The complementary characterizations developed and the proposed statistical model allow the generation or formulation of desired absorbent hydrogels, based on potato starch, poly (vinyl alcohol), and $\mathrm{N}, \mathrm{N}$-methylenebisacrylamide, in technical terms and cost/benefit criteria for specific purposes.

\section{References}

[1] Ullah K, Sohail M, Murtaza G, Khan SA (2018) Int. J. Biol. Macromol 122:538548

[2] Wen N, Jiang B, Wang X, Shang Z, Jiang D, Zhang L, Sun C, Wu Z, Yan H, Liu C, Guo, Z (2020) Chem. Rec 20: 773-792

[3] Rafeeka MR, Rabatb NE, Yahyab WZN, Ghania MA (2019). ChemE 72:175-180

[4] Baghaie S, Khorasani MT, Zarrabi A, Moshtaghian J (2017) J. Biomater. Sci. Polym. Ed 28: 2220-2241

[5] Sirousazar M, Ghanizadeh E, Rezazadeh B, Abbasi-Chianeh V, Kheiri F (2019) J. Environ. Polym. Degrad 27:2842-2852

[6] Anagha B, George D, Maheswari PU, Begum KMS (2019) J. Polym. Environ. 27:2054-2067

[7] Huang M, Hou Y, Li Y, Wang D, Zhang L (2017) Des Monomers Polym 20:505-513

[8] Zulfiqar M, Lee SY, Mafize AA, Adlin N, Abdul M (2020) Polymers 12:430

[9] Bai H, Li Z, Zhang S, Wang W, Dong W (2018) Carbohydr. Polym 200:468-476

[10] Liu P, Chen W, Liu C, Tian M, Liu P (2019) Sci. Rep 9: 1-12, 2019.

[11] Tang X, Alavi S (2011) Carbohydr. Polym 85:7-16

[12] Clerici MTPS, Schmiele M (2018) Starches for Food Application: Chemical, Technological and Health Properties. Campinas, Brazil

[13] Benavent-Gil Y, Rosell CM (2017) Int. J. Biol. Macromol 103:587-595

[14] Saari H, Rayner M, Wahlgren M. (2019) Food Hydrocoll 89:844-855

[15] EL HALAL, Shanise LM et al. (2018) Starch-Stärke 70:1700115

[16] Shafqat A, Tahir A, Mahmood A, Tabinda AB, Yasar A, Pugazhendhi A (2020) 27: 101540 
[17] Arum KS, Prastiti EC, Trisanti PN, Sumarno (2020) AIP 2237:1-6

[18] Biduski B, da Silva WMF, Colussi R, El Halal SLDM, Lim LT, Dias ÁRG, da Rosa Zavareze E (2018) Int. J. Biol. Macromol 113: 443-449

[19] Abdullah HD, Chalimah AS, Primadona I, Hanantyo MHG (2018) IOP Publishing 160:012003

[20] Dankar I, Haddarah A, Omar FEL, Sepulcre F. (2018) Food Chem 260:7-12

[21] Zhang H, Huang X, Jiang J, Shang S, Song Z (2017) RSC Adv 7:42541-42548

[22] Fekete T, Borsa J, Takács E, Wojnárovits L (2014) Radiat. Phys. Chem 118:114119

[23] Kordjazi S, Kamyab K, Hemmatinejad N (2020) Adv. Compos 3:167-176

[24] Hassan A, Bilal M, Niazi K, Hussain A, Farrukh S (2017) J. Polym. Environ 26: $235-243$

[25] Olad A, Doustdar F, Gharekhani H (2018) Carbohydr. Polym 200:516-528

[26] Ghasemzadeh H, Ghanaat F (2014) J. Polym. Res 21:1-14

[27] Singh B, Sharma V (2010) Int. J. Pharm 389:1-2

[28] Braşoveanu, M., Nemţanu, M. R., \& Duţă, D. (2013) Braz. J 30:847-856

[29] Wang L, Wang W, Wang Y, Xiong G, Mei X, Wu W (2018) Int. J. Food Prop $21: 2121-2134$

[30] Reddy BV, Rao GR (2008) Indian J. Pure Appl. Phys 46:611-616

[31] Batool S, Hussain Z, Niazi MBK, Liaqat U, Afzal M (2019) J. Drug Deliv. Sci. Technol 52:403-414

[32] Ahmed A, Niazi MBK, Jahan Z, Samin G, Pervaiz E, Hussain A, Mehran MT (2020) J. Polym. Environ 28: 100-115

[33] Ahmed A, Niazi MBK, Jahan Z, Ahmad T, Hussain A, Pervaiz E, Janjua HA, Hussain Z (2020) Eur. Polym. J 130:109650

[34] OunkaewA, Kasemsiri P, Jetsrisuparb K, Uyama H, Hsu YI, Boonmars T, Artchayasawat A, Knijnenburgd JTN, Chindaprasirt, P (2020) Carbohydr. Polym 248:116767

[35] Pang SC, Chin SF, Tay SH, Tchong FM, Carbohydr. Polym 84: 424-429

[36] Shukla S, Bajpai AK, Kulkarni RA, (2005) J. Appl. Polym. Sci 95:1129-1142

[37] Zhai M, Yoshii F, Kume T, Hashim K, (2002) Carbohydr. Polym 50:295-303

[38] Altaf F, Bilal M, Niazi K, Jahan Z, Ahmad T, Aftab M (2020) J. Polym. Environ 29: $156-174$

[39] Ebadi SV, Fakhrali A, Ranaei-Siadat SO, Gharehaghaji AA, Mazinani S, Dinari 
M, Harati J (2015) RSC 5:42572-42579

[40] Azizi H, Barzin J, Morshedian J, (2007) Express Polym. Lett 1:378-384

[41] Pan Y, Liu L, Zhang Y, Song L, Hu Y, Jiang S, Zhao H (2019) Carbohydr. Polym 206:396-402

[42] Yu C, Tang X, Liu S, Yang Y, Shen X, Gao C, (2018) Int. J. Biol. Macromol 117:1-6,

[43] Sun S, Liu P, Ji N, Hou H, Dong H (2018) Food Hydrocoll 77: 964-975

[44] Guo Y, Zeng X, Lai X, Li H (2018) IEEE T Dielect El In 25:741-748

[45] Hu XQ, Ye DZ, Tang JB, Zhang LJ, Zhang X (2016) RSC Adv 6:13797-13802

[46] Xiang A, Wang H, Liu D, Ma S, Zhang X, Tian H (2018) J. Polym. Eng 38:659_ 665

[47] Liu Y, Wei H, Wang Z, Li Q, Tian N (2018) Polymers 10: 1178

[48] Uto K, Ebara M, Aoyagi T (2014) Int. J. Mol. Sci 15:1511-1524

[49] Ozturk MK, Nergis B, Candan C (2018) IOP Publishing 460:012048

[50] Salcedo J, Contreras K (2017) Agroindustria de productos amiláceos I. Sincelejo, Sucre 